\title{
How to run a National Conference over six time zones during a global pandemic!
}

\begin{abstract}
N eedless to say, the COVID-19 pandemic has impacted all aspects of life one way or another. It has certainly altered business as usual at the Canadian Institute of Forestry/ Institute forestier du Canada (CIF/IFC). Through the unprecedented challenges of the past year, however, the Institute has strived to support the forest sector in innovative ways. For the first time in the Institute's 112-year history, the annual National Conference and Annual General Meeting (AGM) was hosted in a completely virtual format. Both turned out to be remarkably successful, and we share some of our best practices and lessons learned along the way.

For those who may not know, the CIF/IFC's National Conference and
\end{abstract} AGM is an annual event hosted by one of 19 regional CIF/IFC Sections with support from the National office in a different location in Canada each year. The goal is to bring together those interested in forests and forestry, including forest practitioners and professionals, from across Canada and beyond for knowledge sharing and networking. In the spring of 2020, planning for the Conference, to be hosted by the Central Ontario Section in Sault Ste. Marie in September, was already well underway. However, on the advice from public health officials regarding the COVID-19 pandemic, the Institute moved the format to a fully virtual experience that targeted a national audience.

Staff at the National office quickly pivoted to organizing and planning the Institute's first ever virtual Conference and AGM within just four months. This would be an entirely new conference from the one that was to take place in Sault Ste. Marie, complete with a new program and budget, new speakers,

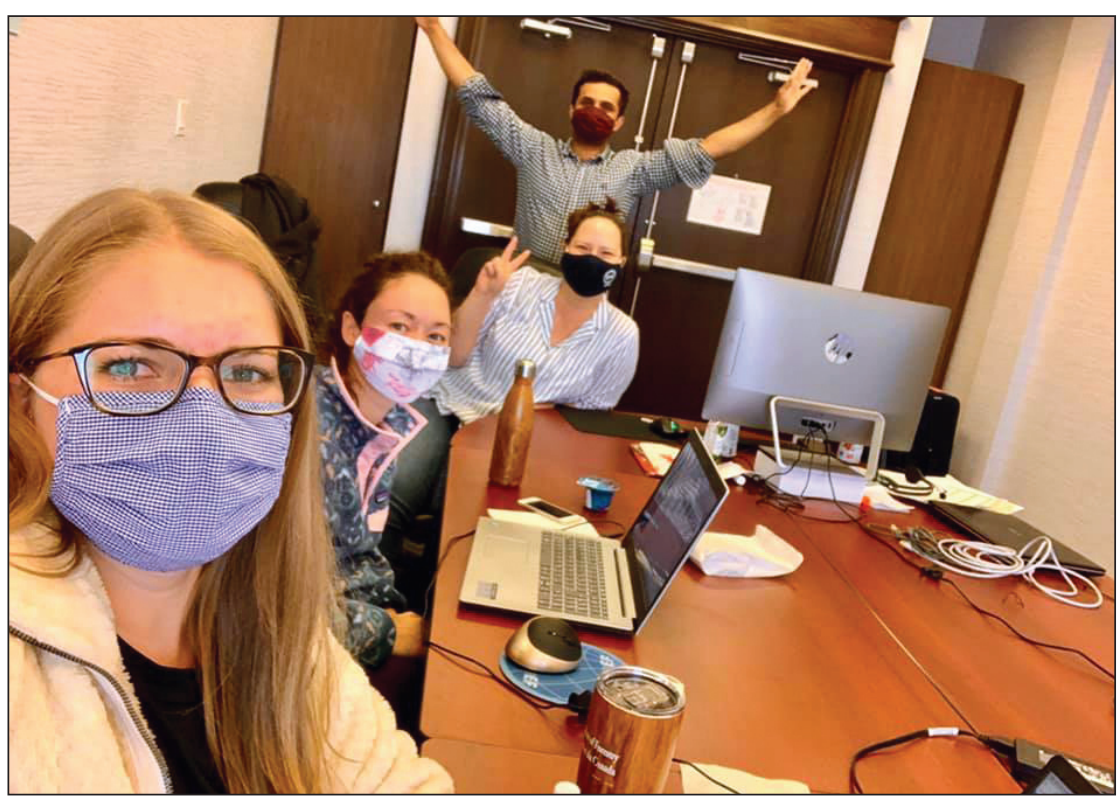

CIF/IFC National Conference command centre The faces behind the screens... CIF/IFC Staff in the virtual Conference "Command Centre", left to right: Jen Dickman, Natasha Machado, Steph Robinson, and Asad Choudhry/Centre de commandement de la conférence nationale de l'IFC/CIF Les personnes derrière l'écran... Le personnel de l'IFC/CIF pour la conférence nationale, de gauche à droite : Jen Dickman, Natasha Machado, Steph Robinson, et Asad Choudhry.

and new technology. The goal was to provide a "normal" conference experience (as much as possible) while bringing together individuals interested in all facets of forests and forestry. Using a digital yet fully interactive platform, the Institute sought to stimulate open dialogue and discussion, knowledge exchange and networking opportunities-key features of "live" CIF/IFC conferences.

The virtual 2020 CIF/IFC National Conference and $112^{\text {th }}$ AGM was hosted online from September 15-17th 2020. The Conference theme, "Diversity and Innovation - Cultivating Resilience in Forestry," was designed to recognize that creative thinking by a diverse and inclusive network will foster the development of the forest sector's capacity to adapt to and recover from adversity. Throughout the Conference proper, six plenary sessions and eight concurrent sessions were hosted with over 50 experts and high-profile speakers weighing in on current forest research, practices, policies, and innovation to support the Conference theme. A special message from the Honourable Seamus O'Regan, Canada's Minister of Natural Resources, formally opened the Confer-ence on day one. In addition to a full Conference program, the Institute also hosted the National Awards Ceremony, the Student Quiz Bowl, the Annual General Meeting, and an evening virtual social, which all took place throughout the 
week. The Conference had a turnout of over 250 delegates from all corners of North America and even abroad.

The CIF/IFC's first virtual Conference and AGM was an undeniable success, but there were numerous challenges and lessons learned along the way. These have been listed below to serve as a best practice guide to others who may be considering holding their own virtual conference or event.

There is no such thing as too much guidance

Assume that all delegates attending a virtual event will need extensive guidance on navigating the technology and platforms. Not doing so may run the risk of alienating certain groups. Prepare tutorials in either a document and/or video format that explain the platform capabilities. Having these types of materials ready in advance of the event is also a great advertising tool.

There can never be too much advertising The CIF/IFC virtual Conference was one of the first of its kind among forest sector organizations. As such, there was some skepticism and doubt about the quality of a virtual conference that may have deterred people from purchasing tickets. A well-advertised and thorough explanation of the platform capabilities may have enticed more people to attend.

Come to terms with the fact that the virtual event will never be as robust as an in-person event

Try to make the best of it by organizing social opportunities and events that allow interaction and networking between delegates, as well as with exhibitors/sponsors.

A virtual event requires just as much organization as an in-person event

There are a lot of moving parts in planning a virtual event and the importance of staying organized cannot be overstated. Depending on the size of the event, a well-balanced team will be required to manage the budget, the speakers, the sponsors, the exhibitors, the event software/technology, ticket sales and registration, advertising, and marketing. Adequate staff/ volunteers and much preparation and organization is needed before, and especially during, the event to ensure that the live event runs smoothly.

\section{Explore event hosting platforms}

There are many options available other than the typical webinar-based platforms. The CIF/IFC invested in a great virtual event platform called Pheedloop that integrated with Zoom webinar. In addition to live-streaming speaker sessions with interactive capabilities, this platform allowed for a "conference experience" by providing networking opportunities for delegates, advertising opportunities for sponsors and virtual exhibitor booth areas.

\section{Practice makes perfect}

Ensure that all speakers and event participants are well prepared for what to expect during the event. Schedule practice sessions in advance of the event to go over the platform technology and event protocols, and to clear up last minute questions.

\section{Create a command centre}

Though the event may be remote, the team facilitating the event should all be in the same location when the event is live. Having the team in one location enables effective management of the event and communication between team members, ensuring the event will run smoothly. Investing in good quality headsets will ensure that there are no distractions when the event goes live.

\section{Incorporate both official languages}

The CIF/IFC is a national organization and as such, recognizes both official languages of Canada. Depending on the size of the event, language translation services can be an expensive endeavour.
Some organizations may not have the budget to incorporate full language translation into their virtual event. There are ways to insert both official languages in an event without incurring extraordinary costs, for example: written material, whether digital or printed, can be in both languages; introductory/closing remarks for the event can be provided in both official languages; and/or create a Conference session that is exclusively one language or the other. Some creative thinking can incorporate both official languages at a reasonable cost.

The CIF/IFC was successful in pivoting from an in-person conference to a virtual one under short timelines and with a modest budget, providing quality forest/forestry-based programming to practitioners and professionals at a very reasonable cost. Moving forward, the CIF/IFC is eager to see the innovative methods that other organizations develop to virtually "meet" under these new circumstances, given that event hosting platforms are continually updating to meet the needs of a society that is transitioning to an online/virtual existence. Perhaps one day soon, events can be hosted in-person again. It will be interesting to see if, in the future, these new methods of meeting virtually will be integrated with in-person events. All in all, the CIF/IFC is proud of what was accomplished during these unprecedented times in 2020.

CIF/IFC National Staff

Canadian Institute of Forestry/ Institut forestier du Canada Mattawa, Ontario Email: media@cif-ifc.org Web: www.CIF-IFC.org 


\section{Comment réaliser une conférence nationale en tenant compte de six fuseaux horaires et d'une épidémie mondiale !}

1 va de soi que la pandémie de la COVID-19 a affecté d'une manière ou d'une autre tous les aspects de la vie. Elle a certainement bouleversé la façon habituelle de gérer les affaires de l'Institut forestier du Canada/Canadian Institute of Forestry (IFC/CIF). Cependant, malgré les défis sans précédents de l'année dernière, l'Institut a cherché à soutenir le secteur forestier au moyen de mesures innovatrices. Pour la première fois au cours des 112 années d'existence de l'Institut, la conférence nationale et l'assemblée générale annuelle (AGA) se sont déroulées d'une manière totalement virtuelle. Les deux événements se sont avérés être un succès et nous souhaitons partager dans ce qui suit les meilleures procédures et les conclusions que nous en avons tirées.

Pour ceux et celles qui l'ignorent peut-être, la conférence nationale et l'AGA de l'IFC/CIF constituent un événement annuel organisé par l'une des 19 sections régionales de l'Institut avec la participation du bureau national. L'objectif visé est de réunir les personnes intéressées par les forêts et la foresterie, notamment les spécialistes des forêts et les forestiers professionnels de tout le Canada et de l'étranger, afin de partager des connaissances et de créer des réseaux de contacts. Au cours du printemps de 2020, la planification de la conférence allait bon train sous la direction de la section Centre de l'Ontario et devait avoir lieu à Sault-Ste-Marie en septembre. Cependant, suite aux avis publiés par les agences de santé publique relativement à la pandémie de la COVID-19, l'Institut a décidé d'utiliser un format virtuel complet accessible partout au Canada.

Le personnel du bureau national a rapidement pris en charge l'organisation et la planification de la toute première conférence nationale et de l'AGA de l'Institut devant se déroulés tout juste quatre mois plus tard. Il s'agissait d'une nouvelle conférence par rapport à celle prévue pour Sault-Ste-Marie, offrant un tout nouveau programme et un budget mis à jour, de nouveaux conférenciers et le tout selon une nou- velle technologie. L'objectif était de présenter un cadre " habituel » de conférence (en autant que possible) tout en réunissant autant de personnes intéressées par tous les aspects de la forêt et de la foresterie. Au moyen d'une plateforme numérique mais totalement interactive, l'Institut cherchait à stimuler le dialogue et les franches discussions, les échanges de connaissances et les opportunités de réseautage- les éléments clés des conférences «normales » de l'Institut.

La conférence nationale 2020 de l'IFC/CIF en format virtuel ainsi que la $112^{\mathrm{e}}$ AGA se sont déroulées du 15 au 17 septembre 2020. Le thème de la conférence, Diversité et Innovation - Cultiver la résilience dans le secteur forestier, a été conçu pour souligner que la pensée créatrice élaborée par un réseau cherchant la diversité et l'inclusion favorisera le développement de la capacité du secteur forestier à s'adapter et à rebondir face à l'adversité. Au cours de la conférence proprement dite, six sessions plénières et huit sessions simultanées ont été offertes et totalisaient plus de 50 experts et conférenciers de premier plan pour discuter des recherches en cours en foresterie, des pratiques courantes, des politiques de l'heure et des innovations offertes dans le cadre du thème de la conférence. L'allocution présentée par l'Honorable Seamus O'Regan, ministre des Ressources naturelles du Canada a officiellement ouvert la conférence. En plus du programme complet de la conférence, l'Institut a procédé également à la cérémonie de remise des prix nationaux, au jeu questionnaire entre les étudiants, à l'assemblée générale annuelle et une activité sociale virtuelle tout au long du programme de trois jours. La conférence a réuni plus de 250 participants en provenance de toute l'Amérique du Nord et même de l'étranger.

Cette première conférence virtuelle et assemblée annuelle de l'IFC/CIF s'est avérée être un succès indéniable, mais de nombreux défis ont dû être surmontés et plusieurs leçons sont à tirer de cette expérience. Celles-ci sont énumé- rées ci-après comme étant les meilleures pratiques à suivre lors de la planification d'une conférence ou d'une activité virtuelle.

Mieux vaut fournir trop d'informations que pas assez

Prenez pour acquis que les participants à une activité virtuelle auront besoin de plus d'informations pour pouvoir accéder à la technologie et aux plateformes. Négliger cet aspect pourrait engendrer un risqué d'aliénation par certains groupes. Prévoyez des tutoriels sous la forme de document ou de vidéo afin d'illustrer les capacités de la plateforme utilisée. Ce type de matériel préparé avant la tenue d'une activité représente également un excellent outil promotionnel.

II ne peut pas y avoir trop de publicité La conférence virtuelle de l'IFC/CIF a été la première de ce genre parmi les organisations du secteur forestier. Dans les faits, un certain niveau de scepticisme et de doute relativement à la qualité offerte par une conférence virtuelle, a pu affecter la décision de s'inscrire pour quelques personnes. La diffusion et des explications approfondies des capacités de la plateforme auraient pu encourager l'inscription d'un plus grand nombre de participants.

Vous devez vous rendre à l'évidence qu'une activité virtuelle ne sera jamais aussi dynamique qu'une activité en présentiel

Essayez de bonifier cet aspect par l'organisation d'activités sociales qui permettent les interactions et le réseautage entre les participants, ainsi qu'avec les exposants et les commanditaires.

Une activité virtuelle requiert autant de planification qu'une activité en présentiel On dénombre de nombreux éléments amovibles lors de la planification d'une activité virtuelle et l'importance de demeurer organisé ne peut qu'être souligné. En fonction de la taille de l'activité, la présence d'une équipe bien équilibrée sera requise pour gérer le budget, 
les conférenciers, les commanditaires, les exposants, le matériel technologique requis par l'activité, la vente des billets et les inscriptions, la publicité et le marketing. Un nombre adéquat d'employés/bénévoles, ainsi qu'une préparation approfondie et une planification détaillée sont requis avant le début de l'activité, et surtout au cours de celle-ci, pour qu'elle se déroule sans anicroche.

\section{Évaluer les plateformes d'hébergement d'activité}

Il existe plusieurs options autres que les plateformes typiques de webinaires. L'Institut s'est procuré une plateforme formidable pour les activités virtuelles dénommée Pheedloop qui combine le webinaire Zoom. En plus des sessions des conférences en direct offrant des possibilités d'interactions, cette plateforme permet de "vivre l'activité » en offrant des possibilités de réseautage entre participants, de publicité pour les commanditaires et de visites des kiosques virtuels des exposants.

C'est en forgeant qu'on devient forgeron Assurez-vous que tous les conférenciers et tous les participants savent à quoi s'attendre de cette activité. Prévoyez des sessions de pratique avant la tenue de l'activité afin d'effectuer un survol de la technologie de la plateforme et du déroulement de l'activité, en plus de répondre aux questions de dernières minutes.
Mettez en place un poste de commandement

Même si l'activité se déroule à distance, l'équipe qui supervise celle-ci devrait être réunie dans un même lieu lors du déroulement de l'activité. Le fait d'avoir l'équipe dans un même lieu permet une gestion efficace de l'activité et la communication entre les membres de l'équipe, assurant un déroulement sans heurt. L'achat d'écouteurs de bonne qualité permettra de minimiser les distractions lors du déroulement de l'activité.

\section{Respecter l'usage des deux langues offi- cielles}

L'IFC/CIF est une organisation nationale et en tant que telle, respecte l'usage des deux langues officielles du Canada. En fonction de la taille de l'activité, les services d'interprétation simultanée peuvent s'avérer dispendieux. Certaines organisations peuvent ne pas avoir les moyens financiers permettant de fournir une interprétation simultanée complète d'une activité virtuelle. Il demeure possible d'utiliser les deux langues officielles au cours d'une activité sans avoir à défrayer des coûts inabordables, par exemple, en fournissant des textes soit par voie numérique ou sous forme de documents imprimés dans les deux langues; les allocutions d'introduction ou de conclusion d'une activité peuvent être traduites; ou encore mettre en place une session de l'activité présentée exclusivement dans l'une ou l'autre langue. Des solutions imaginatives peuvent permettre d'incorporer les deux langues à un coût raisonnable.

L'IFC/CIF a réussi à passer d'une conférence en présentiel à une conférence virtuelle dans un court laps de temps et cela selon un budget modeste, tout en offrant un programme de foresterie de qualité à l'attention spécialistes et des professionnels de la foresterie à un coût très raisonnable. Au cours des prochains mois, l'Institut examinera avec soin les méthodes innovatrices que d'autres organisations utiliseront pour tenir des " rencontres " virtuelles en vertu des obligations sanitaires imposées, étant donné que les plateformes d'hébergement continuent de s'adapter aux besoins d'une société qui est en mode de passer du présentiel au virtuel. Somme toute, l'Institut est fier de ce qui a été réalisé au cours de ces moments sans précédents de 2020.

Le personnel du bureau national de l'IFC/CIF

Institut forestier du Canada/ Canadian Institute of Forestry Mattawa, Ontario

Courriel : media@cif-ifc.org Web: www.CIF-IFC.org

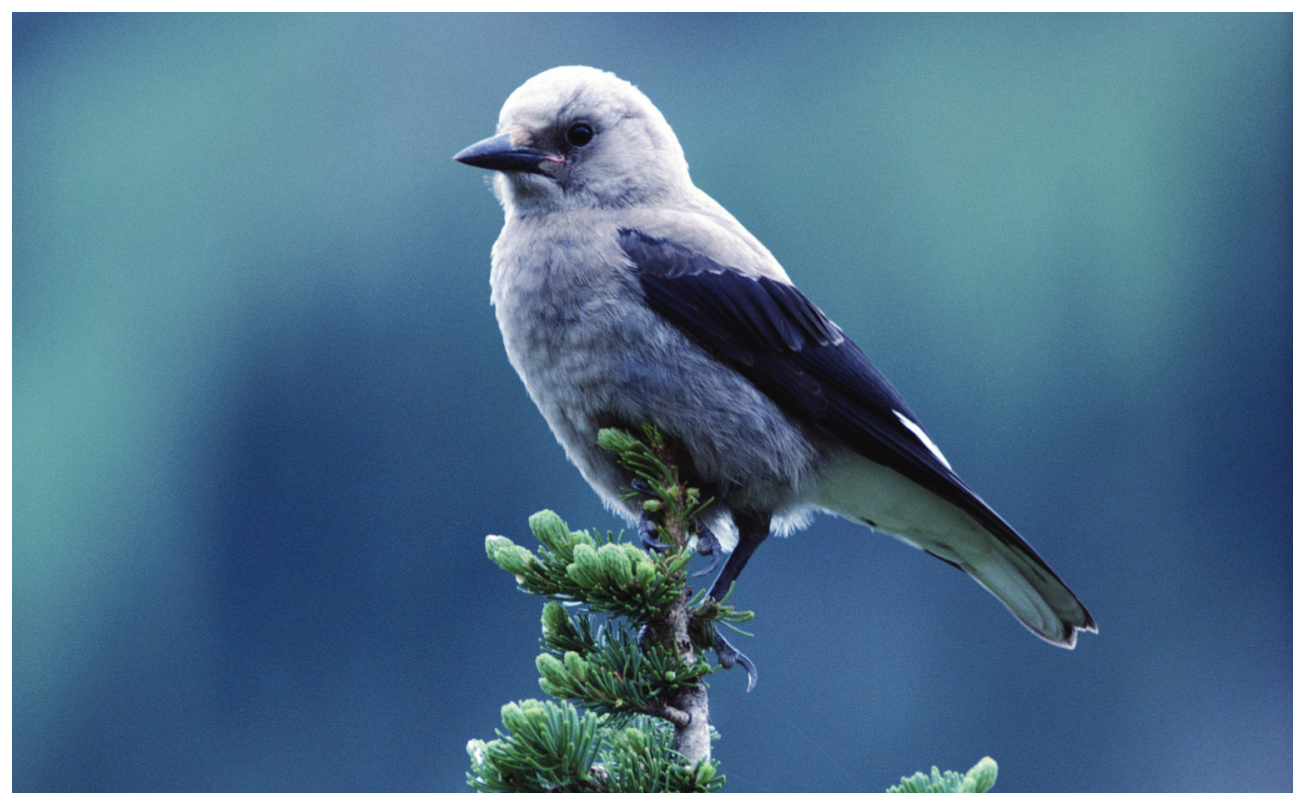

